\title{
A DOR COMO QUINTO SINAL VITAL: UTILIZAÇÃO DA ESCALA DE AVALIAÇÃO POR ENFERMEIROS DE UM HOSPITAL GERAL ${ }^{1}$
}

\author{
Fernanda Hanke Bottega², Rosane Teresinha Fontana ${ }^{3}$
}

\footnotetext{
${ }^{1}$ Artigo originado de um Trabalho de Conclusão do Curso de Graduação Enfermagem da Universidade Regional Integrada do Alto Uruguai e das Missões (URI) - campus Santo Ângelo-RS.

${ }^{2}$ Acadêmica do Curso de Enfermagem da URI - campus Santo Ângelo-RS. Rio Grande do Sul, Brasil. E-mail: fernandabottega@ bol.com.br

${ }^{3}$ Mestre em Enfermagem. Professora do Curso de Enfermagem da URI - campus Santo Ângelo-RS. Rio Grande do Sul, Brasil. E-mail: rfontana@urisan.tche.br
}

\begin{abstract}
RESUMO: Pesquisa descritiva, que objetivou descrever as impressões dos enfermeiros sobre o uso de uma escala visual analógica de avaliação da dor em adultos. Os dados foram coletados por meio de um questionário aplicado a 14 enfermeiros de um hospital e analisados mediante análise temática, resultando em seis categorias: a avaliação da dor e sua importância; a avaliação da dor oportunizando humanização do cuidado; a escala da dor medindo subjetividade; a avaliação da dor oportunizando humanização do cuidado; a aplicação da escala orientando a tomada de decisões e a evolução do cuidado e; a dor como quinto sinal vital. O uso da escala possibilitou que os enfermeiros percebessem a dor como o quinto sinal vital, permitiu-lhes acompanhar a eficácia do cuidado e humanizá-lo. Pode-se inferir que a avaliação da dor por meio de uma escala facilita a tomada de decisões do enfermeiro, favorecendo o cuidado atento às necessidades do paciente.
\end{abstract}

DESCRITORES: Dor. Avaliação em enfermagem. Assistência ao paciente

\section{PAIN AS THE FIFTH VITAL SIGN: USE OF THE ASSESSMENT SCALE BY NURSES IN GENERAL HOSPITAL}

\begin{abstract}
This descriptive study aimed to describe nursing impressions on using a visual analogue scale for assessing pain in adults. Data was collected through a questionnaire administered to 14 nurses at a hospital. Data analysis, using thematic analysis, resulted in six categories: pain assessment and its importance; pain assessment enabling humanization of care; the pain scale measuring subjectivity; pain assessment enabling humanization of care; guiding the implementation of scale-making; the evolution of care, and pain as a fifth vital sign. The use of the scale enabled nurses to perceive pain as the fifth vital sign, as well as allowed them to monitor the effectiveness of care and humanize it. It can be inferred that using a scale in assessing pain facilitates nursing decision-making by encouraging attentive care to patients' needs.
\end{abstract}

DESCRIPTORS: Pain. Nursing assessment. Patient care

\section{EL DOLOR COMO QUINTO SIGNO VITAL: USO DE LA ESCALA DE EVALUACIÓN POR LAS ENFERMERAS EN LOS HOSPITALES GENERALES}

\begin{abstract}
RESUMEN: Es una investigación descriptiva con el objetivo de describir las impresiones de las enfermeras sobre el uso de una escala analógica visual para evaluar el dolor en los adultos. Los datos se recolectaron a través de un cuestionario aplicado a 14 enfermeras en un hospital y se analizaron mediante análisis temático, lo que resulta en seis categorías: la evaluación del dolor y su importancia; la evaluación del dolor para la humanización de la atención; la escala de dolor de medición subjetiva; la aplicación de la escala para orientar la toma de decisiones, y el dolor como un quinto signo vital. El uso de la escala permitió que las enfermeras percibiesen el dolor como el quinto signo vital, les permitió controlar la eficacia de la atención y humanizarla. Se puede inferir que la evaluación del dolor mediante una escala facilita la toma de decisiones del enfermero mediante el fomento de cuidado atento a las necesidades del paciente.
\end{abstract}

DESCRIPTORES: Dolor. Evaluación en enfermería. Atención al paciente 


\section{INTRODUÇÃO}

A dor é uma das principais causas do sofrimento humano, suscitando incapacidades, comprometimento da qualidade de vida e imensuráveis repercussões psicossociais e econômicas, o que a torna um problema de saúde pública. Estudos epidemiológicos, nacionais e internacionais, demonstram que aproximadamente $80 \%$ da procura das pessoas pelos serviços de saúde são motivadas pela dor. A dor crônica acomete 30 a $40 \%$ dos brasileiros e constitui a principal causa de absenteísmo, licenças médicas, aposentadorias por doença, indenizações trabalhistas e baixa produtividade no trabalho. ${ }^{1}$

Definida pela Associação Internacional para o Estudo da Dor (IASP) como "uma experiência sensorial e emocional desagradável que é associada a lesões reais ou potenciais", ${ }^{2: 1}$ a dor é sempre subjetiva. De um modo geral, a dor aguda ou crônica leva a sintomas como alterações nos padrões de sono, apetite e libido; irritabilidade; alterações de energia; diminuição da capacidade de concentração, além de dificuldades em atividades familiares, profissionais e sociais. ${ }^{3}$

A dor é um fenômeno multidimensional, e, caracterizá-la como tal significa observar e avaliar a experiência nas suas várias dimensões, quais sejam: neurofisiológica, pois envolve mecanismos de ativação dos receptores periféricos; psicossocial, considerando a influência emocional positiva e negativa sobre o indivíduo; cognitivocultural, relacionando-a a crenças, significados e comportamentos prévios a dor; comportamental, pois estressores situacionais, de desenvolvimento profissional e pessoal podem exercer influência sobre o limiar da dor; e sensorial, relativas às características semiológicas da mesma. ${ }^{4}$

Isto posto, deve o enfermeiro explorar a queixa de dor, coletar dados sobre fatores agravantes, atenuantes e concomitantes; antecedentes pessoais e familiares, explorar indicativos de desconforto causado pela dor e utilizar-se de instrumentos que podem auxiliar na sua mensuração e avaliação, bem como na qualidade da analgesia. Promover o alívio da dor exige habilidade, conhecimento e acima de tudo compromisso com o cuidado solidário.

A Agência Americana de Pesquisa e Qualidade em Saúde Pública e a Sociedade Americana de Dor a descrevem como o quinto sinal vital, e como tal, deve ser avaliado tão automaticamente quanto os outros sinais vitais do paciente, quais sejam: temperatura, pulso, respiração e pressão arterial. ${ }^{5}$ Como as intervenções para alívio da dor fazem parte do cuidado, fundamental é compreender seu significado e ampliar o campo de saberes acerca da importância de sua mensuração. Para tanto, técnicas que visem avaliar a dor podem ser utilizadas para cuidar de forma humanizada e, medidas diagnósticas inovadoras são estratégias de suporte que contribuem para a atenção às necessidades básicas do indivíduo doente. Neste sentido, avaliar a dor e empenhar medidas para seu alívio, proporcionando conforto e bem estar ao sujeito, podem ser considerados como dispositivos capazes de promover a saúde durante a internação hospitalar ou em cuidados domiciliares.

Em um estudo que teve como objetivo identificar o conhecimento de uma equipe de enfermagem de um hospital em relação à avaliação da dor foi verificado que a mesma não tinha como rotina esta prática e a desconhecia como quinto sinal vital, porém, ao ser educado quanto à importância dessa avaliação, o grupo passou a oferecer um cuidado mais qualificado. Acreditam os autores que, a partir do desenvolvimento da ação educativa proposta, os profissionais compreenderam a subjetividade implícita na queixa de dor, gerando impacto positivo sobre o cuidado de enfermagem, na medida em que sensibilizou os gestores para a implantação do programa de avaliação da dor como quinto sinal vital em todas as unidades do hospital. ${ }^{6}$

Uma grande variedade de estratégias para avaliação da dor pode ser utilizada, sendo que cada modo de avaliação fornece informações qualitativas e quantitativas a respeito da dor. Por ser uma experiência subjetiva, a dor não pode ser mensurada por instrumentos físicos que, usualmente, mensuram o peso corporal, a temperatura, a altura, a pressão sanguínea e o pulso, e ainda não existe um instrumento padrão que permita ao enfermeiro mensurar essa experiência tão complexa e pessoal, porém estão disponíveis algumas escalas que permitem avaliá-la, complementando o processo de análise semiológica do enfermeiro relativo a esta experiência.

É importante ressaltar que a dor deve, sempre, ser avaliada num ambiente clínico, para se empreender, adequadamente, o tratamento ou conduta terapêutica. A eficácia do tratamento e o seu seguimento dependem de uma avaliação e mensuração confiável e válida. Há instrumentos unidimensionais e tridimensionais para a avaliação da dor. Exemplos desses instrumentos são a 
escala de descritores verbais diferenciais, o Questionário McGill de avaliação da dor, a teoria da detecção do sinal e a escala de categoria numérica/ verbal e visual analógica que são frequentemente empregadas em ambientes clínicos, por serem de aplicação fácil e rápida. ${ }^{5}$

Um estudo realizado em uma unidade de terapia intensiva comparou o uso de três escalas de avaliação da dor e verificou que a escala visual analógica não foi um instrumento adequado para pacientes com diminuição da consciência, o que pode estar relacionado com a falta de capacidade para a captação e compreensão, porém foi um instrumento adequado para medir a dor em cerca de dois terços dos pacientes responsivos, com abstração intacta.?

Na avaliação da dor não se deve negligenciar a descrição do paciente sobre o padrão, a intensidade e a natureza da mesma ${ }^{2}$, considerando que, por ser subjetiva, somente o indivíduo pode descrevê-la da forma como é sentida. Escalas de avaliação podem ajudar na eficácia das intervenções, além de respeitar a subjetividade do paciente, pois só ele é capaz de descrever e avaliar com exatidão sua dor; as escalas permitem esta "validação" pelo paciente, o sujeito que vivencia a dor é o expert sobre o padrão, localização, intensidade e natureza da dor, assim como o grau de alívio obtido pela terapia. ${ }^{8}$

Além disso, manifestações como choro, resmungos, gritos e proteção de partes do corpo também podem ser consideradas para tal avaliação. O padrão da dor pode ser avaliado pela descrição do paciente, questionando-o se a dor é constante, intermitente ou breve, e sobre a data e horário do seu início e último episódio. A localização pode ser avaliada solicitando ao paciente que a demonstre num diagrama ou descreva as áreas dolorosas e a intensidade da dor, que pode ser avaliada por meio de uma Escala Visual Analógica (EVA). ${ }^{2}$

Sabe-se que a avaliação da dor ainda é desafiadora, considerando o quadro clínico ou o estágio da doença, a incapacidade do doente em se comunicar e/ ou a urgência iminente. Além disso, ainda há controvérsias sobre o manejo da dor e da analgesia, porém ao se optar por medi-la, tal como outros sinais vitais, as escalas de avaliação são úteis, pois validam a dor pelo paciente. Em muitos casos, após a avaliação da dor, a eficácia e a titulação de analgésicos são aclamadas conforme o padrão desejável. ${ }^{8}$

Enfermeiros devem ter competências e habilidades para avaliar a dor, implementar estra- tégias de alívio da mesma e monitorar a eficácia dessas intervenções. Sendo assim, o objetivo deste estudo foi descrever as impressões dos enfermeiros sobre o uso de uma Escala Visual Analógica, de avaliação da dor em adultos, de modo a sensibilizá-los para o uso destas escalas no cotidiano do cuidado e oportunizar um espaço para reflexão acerca da dor como quinto sinal vital. Como o investigação da dor tem a finalidade de prestar um cuidado mais expressivo e atento às necessidades do paciente, acredita-se que a aplicação de uma escala pode melhorar o processo decisório do enfermeiro quanto às medidas de alívio da mesma, acrescentando dados ao que o enfermeiro já está habituado a realizar.

\section{MÉTODO}

Estudo do tipo descritivo, pois teve como objetivo primário a descrição das características de determinado fenômeno ${ }^{9}$ e de abordagem qualitativa, propiciando campo livre à realidade e à subjetividade dos sujeitos. ${ }^{10}$

O estudo foi realizado no primeiro semestre de 2009, em uma instituição hospitalar da Região Noroeste do estado do Rio Grande do Sul, com 174 leitos. Trata-se de um hospital de referência regional e conta com aproximadamente 400 funcionários, sendo que, deste grupo, em torno de 210 são profissionais de enfermagem distribuídos nas categorias de auxiliar/técnicos em enfermagem e enfermeiros.

Participaram do estudo 14 enfermeiros que trabalham com pacientes adultos do sexo feminino e masculino nos turnos da manhã, tarde e noite em unidades de internação clínica e cirúrgica.

Os dados foram coletados da seguinte maneira: Foi solicitado que os enfermeiros aplicassem a escala e, após seu uso e tomada de decisões, respondessem a um questionário composto por uma pergunta aberta, sobre o qual argüiram com suas próprias palavras ${ }^{10}$ registrando suas impressões sobre o método aplicado. A escala utilizada para a avaliação da dor foi a Escala Visual Analógica, compreendida como uma linha horizontal de 10 cm com as extremidades indicando "ausência de dor" e "a pior dor possível", utilizando-se uma régua e medindo-se a distância entre a marcação do indivíduo, que está sendo avaliado, e o extremo inferior, numa escala em centímetros. ${ }^{2}$

Sabe-se que o uso de escalas apresenta limitações ${ }^{2}$, especialmente a crianças, idosos e pacientes com déficit visual e cognitivo. Há algumas 
restrições quanto ao seu uso em alguns distúrbios cardiovasculares, metabólicos e hormonais, entre outros, que podem alterar a avaliação. Estas limitações, embora não consideradas no estudo, foram socializadas com os enfermeiros, a fim de preservar a eticidade do cuidado durante a pesquisa. Não foi objeto do estudo classificar a dor por especificidade clínica ou argüir acerca destas limitações no processo de mensuração.

A análise temática foi feita a partir da ordenação dos dados coletados, organizando-se os registros obtidos pela entrevista. Mediante leitura exaustiva de todo o conteúdo, procedeu-se à classificação, de onde se extraíram as estruturas de relevância para a determinação dos temas e categorização ${ }^{11}$, de onde emergiram seis categorias: a avaliação da dor e sua importância; a avaliação da dor oportunizando humanização do cuidado; a aplicação da escala orientando a tomada de decisões e a evolução do cuidado; a escala da dor medindo subjetividade; a dor como quinto sinal vital; a formação do enfermeiro para aplicação da escala de avaliação da dor. Por fim, procedeu-se à articulação entre os dados e a literatura, a fim de responder as questões da pesquisa com base nos seus objetivos.

Foram respeitados os preceitos éticos da pesquisa envolvendo seres humanos, estabelecidos pela Resolução $n^{\circ}$ 196/96 do Conselho Nacional de Saúde. ${ }^{12}$ A coleta de dados foi realizada mediante aprovação do estudo pelo comitê de ética da Universidade Regional Integrada do Alto Uruguai e das Missões - campus Santo Ângelo-RS, protocolado sob n ${ }^{\circ}$ 087-04/PPH/08 e da autorização do gestor do hospital em estudo. O Termo de Consentimento Livre e Esclarecido foi assinado pelos sujeitos que se propuseram a participar. Para manter o anonimato dos sujeitos, os enfermeiros foram identificados por números. Pretendeu-se desenvolver a pesquisa com a maior eticidade possível, compartilahndo-se com os enfermeiros a preocupação dos pesquisadores para que a semiologia da dor avaliada fosse observada com muita atenção pelos mesmos, de modo que nenhum paciente fosse exposto a esta avaliação se não estivesse em boas condições físicas e/ou mentais para apontar a intensidade da dor que vivenciava. Da mesma forma, não foi intenção deste estudo questionar a terapêutica utilizada mediante a avaliação da dor.

É válido ressaltar que, como ainda não estava instituída na rotina do hospital a utilização de escalas para avaliação do dor, foi disponibilizado ao enfermeiro uma revisão da literatura sobre a temática, caso o profissional tivesse interesse na leitura. Sabe-se que a sistematização da assistência de enfermagem em todas as suas etapas favorece a avaliação, porém, considerando que o hospital em estudo está em fase de implantação do Processo de Enfermagem em algumas unidades e utiliza parte do processo em outras, a aplicação da escala foi feita mediante a queixa de dor do paciente, um fragmento da sistematização da assistência de enfermagem, todavia era o que se dispunha para desenvolver a pesquisa.

\section{RESULTADOS E DISCUSSÃO}

\section{A avaliação da dor e sua importância}

Quanto à importância da avaliação da dor, observou-se unanimidade dos sujeitos em considerar a relevância de avaliar a intensidade da dor no paciente.

[...] a escala de mensuração da dor é importante no sentido de averiguar a intensidade da dor e até que ponto influencia nas atividades diárias, bem como a eficácia do tratamento e a extensão do problema (E7).

O uso da escala torna visível, mensurável, um sinal que muitas vezes é apenas percebido e registrado de forma incompleta. Ao aplicar a escala da dor, vários questionamentos surgem, partindo daí, uma assistência de enfermagem mais adequada, dentro do que esta dor representa e como se apresenta ao paciente que a sente (E10).

[...] é importante avaliar e considerar o limiar de dor fisiológico para cada indivíduo variável em diferentes ocasiões e influenciado por fatores culturais e psicológicos (E12).

É de grande importância essa mensuração da dor, pois é através dela que o paciente pode descrever ou ser avaliado quanto à exatidão de sua dor (E14).

Cuidar em enfermagem pressupõe, também, estar atento às queixas subjetivas dos pacientes, de modo a intervir no curso do sintoma, possibilitando conforto e bem-estar. Sendo assim, na medida em que se mensura a dor como um sinal vital tem-se parâmetros para estabelecer um bom plano de cuidados, considerando que o cuidado terapêutico deve estar condicionado à intensidade da dor.

Para a deteç̧ão da queixa álgica, sistematizar a avaliação e registrar os dados é fundamental. Dor não identificada e descrita não é tratada. Os dados de avaliação são a base para o diagnóstico etiológico da dor, da prescrição terapêutica e para avaliação da eficácia obtida. ${ }^{13}$ 
A avaliação da dor oportunizando humanização do cuidado

Em relação à humanização no cuidado ao paciente com dor, a maioria dos sujeitos pesquisados percebeu que a partir do momento em que iniciou a aplicação da escala junto aos pacientes, o atendimento tornou-se mais humanizado, como pode ser evidenciado nos depoimentos abaixo:

Vejo a aplicação da escala da dor como uma forma de humanizar o atendimento onde deixamos de olhar para equipamentos e nos voltamos para o paciente, para o que ele relata e está sentindo, damos a eles voz ativa e direito de expressão (E3).

[...] este método é de grande valia no desempenho/ planejamento assistencial para que possamos atribuir um cuidado mais expressivo em relação à patologia e ao paciente como um todo, buscando com isso minimizar o sofrimento/dor do paciente (E9).

Em pacientes que foi utilizado este recurso, foi de grande valia, visto que visa um melhor atendimento ao paciente (E14).

Observou-se que a avaliação da dor possibilitou reflexões acerca de um melhor planejamento da assistência de enfermagem, de modo a oferecer cuidado integral e individualizado para o paciente com dor, possibilitando a implementação de intervenções que minimizem o sofrimento, tornando $\mathrm{o}$ atendimento mais humanizado e atento às necessidades. A humanização no atendimento ao paciente é uma estratégia que deve estar presente nos diversos níveis de atenção à saúde. Sabe-se que a política da humanização no atendimento prevê mudança nos modelos de atenção e gestão dos processos de trabalho, tendo como foco as necessidades dos usuários e a produção de saúde, bem como a identificação das necessidades, desejos e interesses dos mesmos. Considerar a pessoa não meramente um corpo biológico, numa visão reducionista, pura e simplesmente, é um desafio. Urge que se tenha uma visão holística, multi, inter e transdisciplinar do ser humano como um todo uno, pois ser gente é possuir corpo físico, mental e espiritual. "Quem cuida e se deixa tocar pelo sofrimento humano do outro torna-se um radar de alta sensibilidade, se humaniza no processo e, para além do conhecimento científico, tem a preciosa chance e o privilégio de crescer em sabedoria." $14: 12$

Os profissionais da saúde, ao oferecer alívio efetivo à dor, o que deve ser feito de acordo com um julgamento apropriado e com as abordagens mais avançadas disponíveis, humanizam o cuida- do na medida em que consideram a subjetividade do sujeito e a satisfação de suas necessidades. O alívio da dor e dos sintomas da doença é uma contribuição importante para a qualidade de vida do paciente, contribuindo para apressar a recuperação, além de outros benefícios. ${ }^{14}$

\section{A aplicação da escala orientando a tomada de decisões e a evolução do cuidado}

Observou-se que grande parte dos entrevistados acreditou que entender melhor a intensidade da dor facilita a tomada de decisões, bem como o acompanhamento da eficácia do cuidado prestado.

[a escala de avaliação da dor] [...] é um instrumento de suma importância e sua aplicabilidade nos norteia a tomar decisões importantes no que se refere à dor do paciente (E4).

Com a mensuração da dor é possível determinar se um tratamento está agindo positivamente sobre o paciente (E5).

Esta escala é importante no sentido de acompanhar a evolução da intensificação da dor e até que ponto o tratamento está beneficiando o paciente, ou como anda a evolução e a intensificação da doença que torna-se mais aguda interferindo na rotina e causando privações que possam influenciar na qualidade de vida do cliente (E7).

É uma ferramenta que nos proporciona com maior exatidão quantificar a dor que o nosso cliente está sentindo e repensar muitas vezes se a terapêutica que está sendo utilizada realmente está sendo eficaz para o tratamento daquele indivíduo (E8).

O enfermeiro, como profissional da área de saúde, continuamente necessita tomar decisões relativas à assistência de enfermagem a ser prestada em sua unidade. O processo decisório é definido como análise e escolha, entre várias alternativas disponíveis, da ação que o profissional adotará, o que envolve avaliação criteriosa e reflexão.

A tomada de decisão envolve fenômenos tanto individuais quanto sociais, e deve incluir a avaliação dos recursos disponíveis, dos conhecimentos que o enfermeiro possui ${ }^{15}$, e processar-se em conexão com as percepções e desejos do paciente a fim de que ele possa participar.

A partir disso, a eficácia do tratamento e a sua continuidade dependem de uma avaliação e mensuração da dor confiável e válida, ${ }^{3}$ o que pressupõe tomada de decisões a partir de uma criteriosa avaliação, com a participação do pa- 
ciente, o que é fundamental no curso da atividade gerencial e cuidadora do enfermeiro. A tomada de decisões embasada no saber científico e na ética confere credibilidade, eficiência e eficácia no trabalho do enfermeiro.

\section{A escala da dor medindo subjetividade}

Embora adeptos à avaliação da dor, quase a totalidade dos enfermeiros participantes entende que a dor é uma experiência subjetiva, o que torna o sujeito protagonista de seu próprio diagnóstico, considerando-se que a vivência da dor é única e individual. Cabe ao enfermeiro respeitar esta condição, interpretar e intervir.

[...] cada ser humano apresenta um limiar diferente no que se relaciona a dor, não podendo nunca compararmos situações e pacientes e sim trabalharmos a questão dor no mesmo (E1).

[...] a grande dificuldade para avaliação da dor se dá por esta mensuração ser de caráter subjetivo, individual e principalmente sentida pelo outro, tendo este um grau de sensibilidade e suportabilidade próprio e único, colocando com isso o profissional da saúde não como mensurador da dor, mas sim como interpretador dessa mensura (E3).

Sendo a dor subjetiva, o profissional deve acreditar no paciente quando este verbaliza sentir dor, devendo-se avaliar aspectos físicos, mentais e emocionais (E5).

Identificar sinais sugestivos de dor contribui para a terapêutica. Esta é uma tarefa que pode gerar dificuldades, visto que pacientes e profissionais podem ter concepções diferentes da dor. Esta pode apresentar-se através de diversas maneiras, tais como: choro, gemido, alterações dos sinais vitais, agitação, tremor ou comportamento verbal. Porém, a ausência dos sinais citados não significa ausência de dor. Alguns pacientes podem adaptar-se à dor, através do desenvolvimento de um elevado autocontrole, suprimindo os sinais de sofrimento, ou apenas permanecendo prostrados ou mais quietos que o habitual, devido ao esgotamento físico e mental causados pela doença, ${ }^{16} \mathrm{o}$ que não impede a sua avaliação.

\section{A dor como quinto sinal vital}

Grande parte dos sujeitos entrevistados identificou a dor como quinto sinal vital, assim devendo ser avaliado, registrado e tratado regularmente como os outros parâmetros fisiológicos, embora não o faça habitualmente. Atualmente, a dor é considerada um sinal vital tão importante quanto os outros (temperatura, pulso, pressão arterial e respiração) e deve sempre ser avaliada num ambiente clínico. ${ }^{3}$

A partir disso, uma alteração expressiva relacionada à intensidade da dor e registrada no prontuário do paciente pode ser utilizada para planejarem-se ações apropriadas ao alivio da mesma. Pode ser significativo, para aumentar a eficácia do método, utilizar a escala de forma sistemática e regular e, embora seja necessário o uso de outros mecanismos clínicos para detectar e avaliar o fenômeno doloroso, tais como observar alterações de comportamento e/ou fisiológicas e, a escala apresente algumas limitações em função da idade e das interferências clínicas características de determinados processos patológicos, aferir a partir de uma escala confere uma propriedade objetiva a um fenômeno subjetivo.

Considero a dor o quinto sinal vital e a julgo tão importante quanto os demais sinais vitais, pois conforme a sua intensidade, pode trazer angústia e incapacidade para o indivíduo, bem como mudar sua fisionomia, maneira de pensar e agir, no meu ver, influenciando diretamente nos valores de outros sinais vitais como $P A, F C$ e FR (E3).

Alguns profissionais ainda não se sensibilizaram sobre a importância de vislumbrar a dor como um quinto sinal vital ou não fazem a mensuração da dor do paciente no momento da entrevista. Disponibilizam, nos seus registros, apenas informações relativas aos outros quatro sinais vitais já incorporados na prática, ${ }^{17}$ o que é lamentável; a avaliação da dor é tão importante quanto os outros sinais, considerando que é difícil prescreverem-se cuidados sem ter um parâmetro para definir condutas de alívio e bem estar ao paciente.

Um estudo realizado em Tubarão-SC, em 2008, que teve como objetivo avaliar a percepção e conhecimento de farmacêuticos, médicos e enfermeiros em relação ao tema dor, assim como a preocupação com a mesma e com o conforto do paciente, evidenciou que, embora demonstrando maior conhecimento das escalas sobre os demais profissionais, metade dos enfermeiros pesquisados não citou ou desconhece as escalas para mensurar a dor. ${ }^{18}$ Em estudo semelhante ${ }^{19}$ realizado no estado de New Hampshire - USA, foi verificado que dentre as categorias profissionais pesquisadas, os enfermeiros tem mais conhecimento a respeito da avaliação da dor, sobre outros profissionais, o que nos faz refletir acerca da formação acadêmica brasileira sobre a temática, que parece ter avançado, porém lentamente. 


\section{A formação do enfermeiro para a aplicação da escala de avaliação da dor}

Embora não tenha sido amplamente referenciado, um número significativo de enfermeiros salientou a importância da formação, do preparo científico para aplicação da escala de avaliação da dor. Considerando-se que tal temática ainda é recente na literatura científica, esta preocupação se justifica.

[a aplicação da escala de avaliação da dor] [...] foi uma maneira de melhor atendermos as necessidades dos pacientes desde que [...] o profissional deverá estar treinado e capacitado para poder avaliar a dor do paciente (E1).

Vejo a escala da dor como um bom instrumento para sua avaliação, mas percebo que a mesma deve ser acompanhada por um grau de experiência e conhecimento por parte do profissional, grau este que só adquirimos através do tempo e aplicação da mesma, pois como cada indivíduo é único, a mesma deve ser adaptada a cada situação, conforme o paciente e sua patologia (E3).

Creio que com o passar dos dias [aprende-se a escala] [...] a rotina ficou mais eficaz, pois todas as experiências novas são bem-vindas, é somente com a prática que vamos obter êxito (E14).

O processo que envolve o ensino-aprendizagem para o desenvolvimento de competências e habilidades, são construções iniciadas na graduação e ampliadas ao longo da prática profissional e permitem apreender tecnologias de cuidado necessárias à complexa dimensão social da enfermagem, qual seja melhorar a qualidade de vida dos usuários dos serviços de saúde. É exigido dos profissionais o compromisso com a atualização, de modo a oferecer alternativas aos sujeitos ao alívio da dor, do sofrimento, das injúrias e iniqüidades, seja no âmbito hospitalar ou comunitário. A falta de conhecimento é um desafio para o cuidar em enfermagem; a educação no lócus do trabalho e a pesquisa, podem ser mecanismos capazes de promover a construção e reconstrução de saberes .

Quando foi pensada uma estratégia para melhor compreensão e avaliação da dor nos pacientes, nos Estados Unidos, na década de 90, enfermeiros foram mobilizados e educados para que avaliassem a dor utilizando escalas de mensuração e registrassem o resultado no prontuário assim como feito aos outros sinais vitais; como um quinto sinal vital. ${ }^{17}$

É válido salientar que, da mesma forma que todo profissional de saúde deve, frequentemente, atualizar seus conhecimentos pela educação per- manente em saúde, como uma estratégia para responder a esta necessidade, um dispositivo para melhor prestação do cuidado pode ser a sua sistematização. Assim, mais ampla e cientificamente consegue-se abordar este sintoma, o que auxilia, efetivamente, na terapêutica.

Melhorias na qualidade da avaliação da dor exigem melhorias de comunicação, em especial do uso de tecnologias de informação, de educação, de discussões e mudanças organizacionais e multidisciplinares. ${ }^{17}$

\section{CONSIDERAÇÕES FINAIS}

A partir da realização desta pesquisa observou-se que a aplicação da escala de avaliação da dor é uma maneira de melhor interpretar e entender a dor do paciente, facilitando o planejamento da assistência e a tomada de decisões do enfermeiro, bem como o acompanhamento da eficácia do tratamento, tornando $\mathrm{o}$ atendimento mais humanizado e atento às necessidades do paciente.

Constatou-se que os enfermeiros estão sensibilizados quanto à importância da aplicação da escala para a avaliação da intensidade da dor, $\mathrm{o}$ que possibilita um atendimento integral e individualizado para o paciente com dor. Embora sendo considerada por todos os sujeitos pesquisados como uma experiência subjetiva, o que dificulta a sua aferição, é necessário avaliar a dor regularmente e o uso das escalas é uma forma eficaz, na concepção dos enfermeiros.

Sendo a dor considerada o quinto sinal vital, este estudo tinha a pretensão de provocar uma reflexão aos enfermeiros acerca deste tema, o que, a partir de suas falas, acredita-se ter conseguido. Os enfermeiros observaram, avaliaram, registraram e, mesmo que parcialmente, sistematizaram o cuidado.

O estudo, mediante observação subjetiva do pesquisador, ofereceu, também, elementos para que o enfermeiro refletisse sobre a importância da sistematização do cuidado, numa lógica que alia organização do tempo e satisfação das necessidades. A inexistência da sistematização do cuidado foi uma limitação importante para este estudo, sobre as quais a sobrecarga de trabalho é uma realidade a ser considerada.

Além disso, cursos de formação, de aperfeiçoamento ou de atualização devem ser incentivados para a aculturação desta proposta, e, que este modelo de cuidado possa estar presente nos currí- 
culos de graduação e nas práticas de ensino clínico para que, a partir disso, construam-se saberes que possibilitem a viabilização desta prática.

\section{REFERÊNCIAS}

1. Ministério da Saúde (BR). Ato Portaria No 19/GM de 03 de janeiro de 2002. [acesso 2009 Ago 17]. Disponível em: http://dtr2001.saude.gov.br/sas/ PORTARIAS/Port2002

2. Sociedade Brasileira para o Estudo da Dor (SBED). Hospital sem dor diretrizes para implantação da dor como $5^{\circ}$ sinal vital [acesso 2009 Jul 20]. Disponível em: http://www.dor.org.br/profissionais/5_sinal_ vital.asp

3. Kreling MCGD, Cruz DALM, Pimenta CAM. Prevalência de dor crônica em adultos. Rev Bras Enferm. 2006 Ago; 59(4):509-13.

4. Posso MBS. Semiologia e semiotécnica de enfermagem. São Paulo (SP): Ateneu; 2004.

5. Sousa FAEF. Dor: o quinto sinal vital. Rev Latino-am Enfermagem. 2002; Mai-Jun; 10(3):446-7

6. Pedroso RA, Celich KLS. Dor: quinto sinal vital, um desafio para o cuidar em enfermagem. Texto Contexto Enferm. 2006 Abr-Jun; 15(2):270-6.

7. Ahlers SJ, Van Gulik L, Van der Veen AM, Van Dongen HP, Bruins P, Belitser SV, et al. Comparison of different pain scoring systems in critically ill patients in a general ICU. Crit Care [online]. $2008 \mathrm{Feb}$ [acess 2009 Agu 5]; 12(1):R15. Available at: http:/ / ccforum.com/content/12/1/R15

8. Skrobik Y. Pain may be inevitable; inadequate management is not. Crit Care [online]. 2008 [acess 2009 Mai 03]; 12(2):142. Available at: http:// ccforum.com/content/12/2/142

9. Gil AC. Métodos e técnicas de pesquisa social. $4^{\mathrm{a}} \mathrm{ed}$. São Paulo (SP): Atlas; 1995.

10. Polit DF, Hungler BP. Fundamentos de pesquisa em enfermagem. $3^{\text {a }}$ ed. Porto Alegre (RS): Artes Médicas; 1994.

11. Minayo MCS. O desafio do conhecimento: pesquisa qualitativa em saúde; $11^{\mathrm{a}}$ ed. São Paulo (SP): Hucitec; 2008.
12. Ministério da Saúde (BR), Conselho Nacional de Saúde, Comissão Nacional de Ética em Pesquisa. Resolução No 196 de 10 de outubro de 1996: diretrizes e normas regulamentadoras de pesquisa envolvendo seres humanos. Brasília (DF): MS; 1996.

13. Pessini L, Bertachini L. Humanização e cuidados paliativos. $2^{\mathrm{a}}$ ed. São Paulo (SP): Loyola; 2004.

14. Pessini L. Humanização da dor e sofrimento humanos no contexto hospitalar. Bioética 2002; 10(2):51-72.

15. Campos DCF, Graveto JMGN. Papel do enfermeiro e envolvimento do cliente no processo de tomada de decisão clínica. Rev Latino-am Enfermagem. 2009 Nov-Dez; 17(6):1065-70.

16. Tulli ACP, Pinheiro CSC, Teixeira SZ. Dor oncológica: os cuidados de enfermagem [online]. São Paulo (SP): Sociedade Brasileira de Cancerologia; 2007 [acesso 2009 Abr 2]. Disponível em: http:/ / www.rsbcancer. com.br/rsbc/7Suplemento.asp

17. Mularski RA, White-Chu F, Overbay D, Miller L, Asch SM, Ganzini L. Measuring pain as the 5th vital sign does not improve quality of pain management. J Gen Intern Med [online]. 2006 Jun [acess 2009 Jun 12]; 21(6):607-12. Available at: http://www.ingentaconnect.com/content/bsc/ jgi/2006/00000021/00000006/art00022

18. Kulkamp IC, Barbosa CG, Bianchini KC. Percepção de profissionais da saúde sobre aspectos relacionados à dor e utilização de opióides: um estudo qualitativo. Ciênc Saúde Colet. 2008; 13(1):721-31.

19. Furtsenberg CT, Ahles TA, Whedon MB, Pierce KL, Dolan M, Roberts L, Silberfarb PM. Knowledge and attitudes of health-care providers toward cancer pain management: a comparison of physicians, nurses, and pharmacists in the State of New Hampshire. J Pain Symptom Manage [online] 1998 [acess 2009 Ago 17]; 15(6):335-49. Available at: http://www.sciencedirect.com/science? $\mathrm{ob}=$ ArticleURL\&_udi=B6T8R-3TK775T $-3 \& \_$ user $=687353 \&$ _coverDate $=06 \% 2 \mathrm{~F} 30 \% 2 \mathrm{~F} 1998 \&$ _ $\mathrm{rdoc}=1 \& \_\mathrm{fmt}=$ high\&_orig $=$ search\&_sort $=\mathrm{d} \&$ _ docanchor $=\&$ view $=c \& \_$acct $=C 000037882 \&$ version $=1 \& \_$urlVersion $=0 \& \_$userid $=687353 \& \mathrm{md} 5$ $=$ b67a22491ec69cf1a6d316aa6fe8f9a9 